Rev. Biol. Neotrop. 5(2): 33-39, 2008

\title{
FEITOS DO HABITAT E DA SAZONALIDADE NA DISTRIBUIÇÃo DE INSETOS galhadores na Serra dos Pireneus, Goiás, Brasil
}

\author{
Walter Santos de Araújo \\ Benedito Baptista dos Santos
}

Laboratório de Entomologia, Departamento de Ecologia, Instituto de Ciências Biológicas, Universidade Federal de Goiás, Campus II, Caixa Postal 131, 74001-970, Goiânia, Goiás, Brasil; e-mail: walterbioaraujo@yahoo.com.br; bbsantos@icb.ufg.br

\begin{abstract}
Resumo: A hipótese do estresse higrotermal prediz gradiente de diversidade no sentido cerrado $\rightarrow$ mata, evidenciando maior preferência dos galhadores por ambientes xéricos. Entretanto, alguns trabalhos têm apontado que outros fatores são mais importantes em escalas locais, como a sazonalidade. Desse modo, os objetivos deste estudo foram: testar a hipótese do estresse higrotermal e verificar se existem efeitos da sazonalidade nos padrões de distribuição dos insetos galhadores. O estudo foi conduzido entre setembro de 2007 e junho de 2008 no Parque Estadual da Serra dos Pireneus, em Pirenópolis, Goiás. Durante o período, foram realizadas amostragens de galhas em um fragmento de vegetação xérica (Cerrado sensu stricto) e mésica (mata semidecídua), através de transectos amostrados por 1 h e 30 min. Os morfotipos de galhas coletados foram encaminhados ao laboratório de Entomologia da Universidade Federal de Goiás para verificação dos insetos galhadores. Não foram observadas diferenças significativas entre a riqueza média de galhas no Cerrado e na mata. Por outro lado, a riqueza de galhas foi estatisticamente diferente quando comparados os números de morfotipos nas estações seca e chuvosa. Os resultados do presente estudo apontam que padrões sazonais podem ser mais determinantes na diversidade de insetos galhadores do que o tipo fitofisionômico.
\end{abstract}

Palavras-chave: Estresse higrotermal, galhas, sazonalidade, tipo fitofisionômico.

AвstRACt: The hypothesis of higrothermal stress predicts a gradient of diversity in the direction Cerrado $\rightarrow$ forest, showing a higher preference of galling insects for xeric environments. However, some studies have pointed out that other factors are more important at local scales, such as seasonality. Thus, the objectives of this study were: test the hypothesis of higrothermal stress and investigate whether there are effects of seasonality in the patterns of distribution of galling insects. The study was carried out from September 2007 to June 2008 at the Parque Estadual da Serra dos Pireneus, in Pirenópolis, Goiás. During this period, samples of galls were collected within a fragment of xeric (Cerrado sensu stricto) and mesic (semideciduous forest) vegetation through transects sampled for $1 \mathrm{~h}$ and $30 \mathrm{~min}$. The gall morphotypes collected were sent to the laboratory of Entomology of the Universidade Federal de Goiás to detect the galling insects. No significant differences were observed between the average richness of galls in the Cerrado and forest. On the other hand, the richness of galls was statistically different comparing the numbers of morphotypes in the dry and rainy seasons. The results of this study indicate that seasonal patterns can play a more decisive role in the diversity of galling insects than the phytophysiognomic type.

KEY wORDs: Higrothermal stress, galls, seasonality, phytophysiognomic type.

\section{INTRODUÇÃo}

I nsetos galhadores são herbívoros bastante especializados que contornaram as dificuldades de proteção e obtenção de alimentos com o desenvolvimento de galhas (Stone \& Schönrogge, 2003). Alguns estudos têm buscado fatores que determinem os padrões de distribuição desses insetos no mundo (Fernandes \& Price, 1988; Price et al., 1998) e no Cerrado (Gonçalves-Alvim \& Fernandes, 2001). Desse modo, várias hipóteses foram propostas para explicar esses gradientes de diversidade (Fleck \& Fonseca, 2007; Mendonça, 2007). 
A hipótese do estresse higrotermal, proposta por Fernandes \& Price (1988), prediz que a riqueza de galhadores está positivamente relacionada com o estresse nutricional do solo (deficiência de água e nutrientes). Estudos comparativos da riqueza de galhas entre diferentes habitats corroboram essa hipótese, demonstrando a existência de gradiente de diversidade no sentido Cerrado $\rightarrow$ mata (Araújo et al., 2007; Fernandes \& Price, 1988; Fernandes et al., 1995; Gonçalves-Alvim \& Fernandes, 2001).

Porém, em outros trabalhos foram encontrados padrões que contrariam a hipótese do estresse higrotermal (Ferreira et al., 2007; Veldtman \& McGeoch, 2003). Em alguns desses estudos, os resultados apontam maior riqueza de insetos galhadores em habitats mésicos (Veldtman \& McGeoch, 2003) ou simplesmente não demonstram diferenças entre os ambientes (Ferreira et al., 2007). Segundo Veldtman \& McGeoch (2003), fatores como o estresse higrotermal e a presença ou ausência de esclerofilia podem ser importantes preditores de padrões de riqueza globais para os galhadores, enquanto outros fatores podem ser mais importantes em escalas locais ou regionais, tais como a composição da comunidade de plantas (CuevasReyes et al., 2004) e as variações nos padrões sazonais (Dalbem \& Mendonça, 2006).

Estudos sobre a influência da sazonalidade na distribuição de insetos herbívoros de vida livre são comuns na literatura (Kasenene \& Roininen, 1999; Wolda, 1988), mas trabalhos que abordam a diversidade de insetos galhadores são escassos (Dalbem \& Mendonça, 2006). Algumas pesquisas apontam que flutuações sazonais não interferem na distribuição dos insetos galhadores (Fernandes et al., 1995). Entretanto, resultados recentes sugerem que a estação climática pode ser um fator determinante na riqueza desses insetos (Araújo \& Santos, 2009; Dalbem \& Mendonça, 2006). Desse modo, os objetivos deste estudo foram: testar a hipótese do estresse higrotermal, que prediz maior riqueza de galhas em ambientes xéricos e verificar se existem efeitos da sazonalidade nos padrões de riqueza dos insetos galhadores.

\section{Material e Métodos}

\section{ÁREA DE ESTUDO}

Oestudo foi realizado no Parque Estadual da Serra dos Pireneus, localizado entre os municípios de Pirenópolis e Cocalzinho de Goiás, a cerca de $18 \mathrm{~km}$ de Pirenópolis $\left(15^{\circ} 48^{\prime} 18,1^{\prime \prime} \mathrm{S}\right.$ $48^{\circ} 52^{\prime} 53,2^{\prime \prime} \mathrm{W}$, Figura 1). A área do parque é de 2.833,26 ha e compreende regiões de Cerrado sensu stricto, Cerrado rupestre, mata de galeria e mata semidecídua. O clima da região, segundo a classificação de Köppen, é do tipo Aw, com estação seca (de abril a setembro) e estação chuvosa (de outubro a março) bem definidas (Ribeiro \& Walter, 1998).

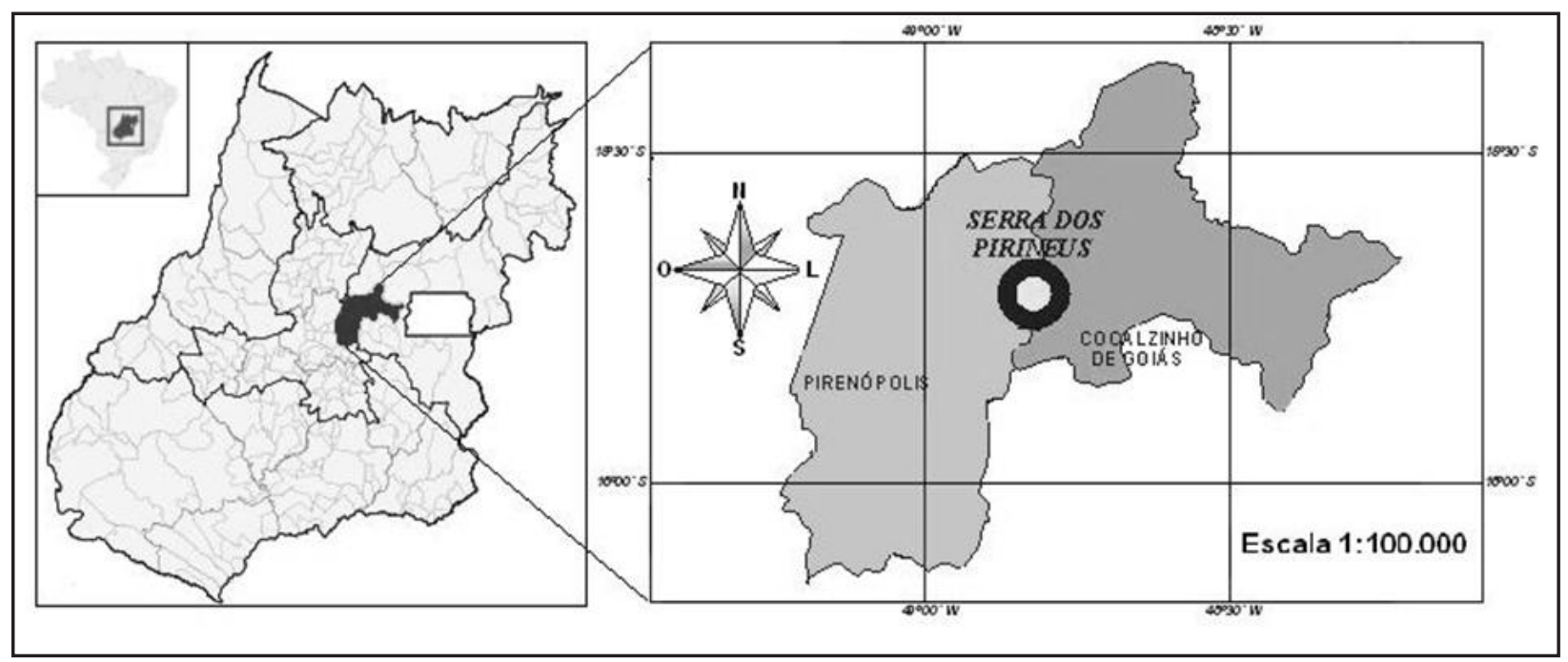

Figura 1 - Localização do Parque Estadual da Serra dos Pireneus, entre os municípios de Pirenópolis e Cocalzinho de Goiás, Goiás, Brasil. 


\section{Amostragem}

Para testar a hipótese do estresse nutricional, no período entre setembro de 2007 e junho de 2008 foram realizadas amostragens em duas fitofisionomias de Cerrado, sendo uma xérica (Cerrado sensu stricto, S 1548'19,2", W 48 52'19,2", elevação de $1.334 \mathrm{~m})$ e outra mésica (mata semidecídua, 15²47'34,4"S $48^{\circ} 50^{\prime} 16,3^{\prime \prime} \mathrm{W}$, elevação de $\left.1.294 \mathrm{~m}\right)$. A metodologia utilizada consistiu em amostragens ao longo de um transecto em cada fitofisionomia, com número de amostradores fixo e tempo de amostragem determinado (Dalbem \& Mendonça, 2006). Cada transecto foi amostrado por dois amostradores durante $1 \mathrm{~h}$ e $30 \mathrm{~min}$, duas vezes no período chuvoso e duas no período seco. A primeira amostragem foi realizada no final da estação seca, a segunda e a terceira no início e no final da estação chuvosa e a quarta no início da estação seca. As amostragens foram realizadas em transectos fixos, de modo que foi possível acompanhar a variação da diversidade de galhas ao longo do gradiente sazonal. Todos os morfotipos de galhas encontrados nos dois habitats foram registrados, caracterizados, fotografados e encaminhados ao laboratório, conforme Araújo et al.(2007).

\section{ANÁLISES ESTATÍSTICAS}

Para verificar a medida de suficiência amostral, foram utilizados os estimadores de riqueza Chao 1 e Jack-knife de primeira ordem
(Jack-knife 1), calculados a partir do programa BioDiversity Pro. As variações da riqueza de morfotipos de galhas entre os habitats xérico e mésico e entre os períodos de seca e chuva foram comparadas usando testes de comparação de médias (Teste t de Student). Análises de variância (ANOVA one-way) foram utilizadas para testar a flutuação da riqueza de galhas entre as diferentes épocas de amostragem. Todas as análises foram realizadas empregando o programa Statistica 7.1.

\section{Resultados}

Foram amostrados 47 morfotipos de galhas no Cerrado e 53 na mata. De acordo com os estimadores, a riqueza de espécies variou entre 47 (Chao 1) e 66 (Jack-knife 1) para o Cerrado e entre 52 (Chao 1) e 79 (Jack-knife 1) para a mata semidecídua.

Não foram observadas diferenças significativas entre a riqueza média de galhas no Cerrado e na mata (Teste $\mathrm{T}: \mathrm{t}=0,17, \mathrm{~N}=8, \mathrm{p}=$ 0,86, Figura 2). No Cerrado, o número médio de galhas foi de 22 (desvio padrão +0 ) na estação seca e de 17,5 (desvio padrão $+2,5$ ) na estação chuvosa, enquanto na mata, foi de 23 (desvio padrão + 2) na estação seca e de 15,5 (desvio padrão $+0,5$ ) na estação chuvosa.

Por outro lado, a riqueza de galhas foi estatisticamente diferente quando comparados os números de morfotipos nas estações seca e chuvosa (Test T: $\mathrm{t}=4,07, \mathrm{~N}=8, \mathrm{p}<0,01$ ). No período seco, foi observado maior núme-

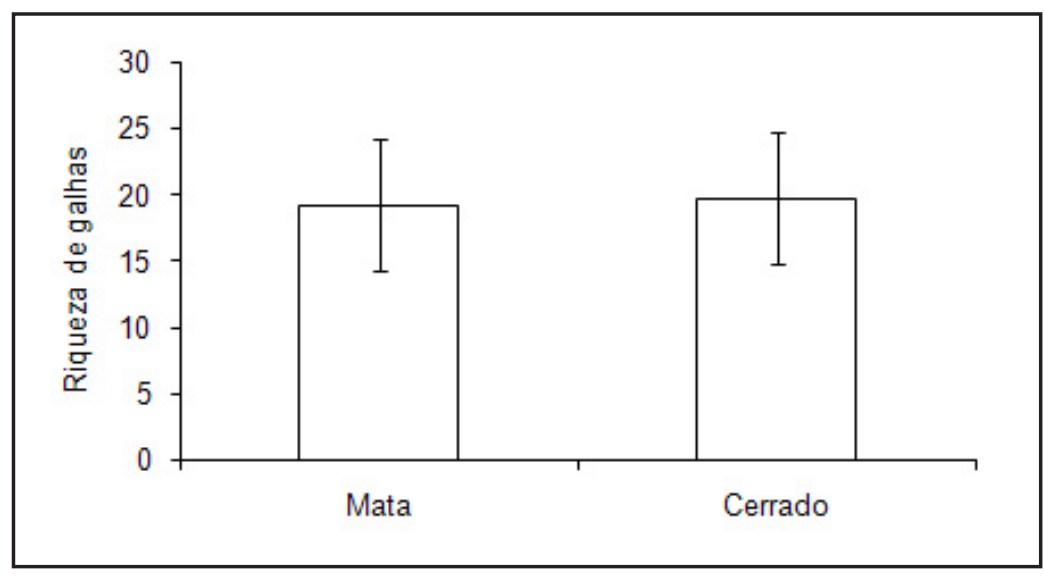

Figura 2 - Distribuição dos morfotipos de galhas em habitats de Cerrado (média e desvio padrão) sensu stricto (xérico) e mata semidecídua (mésico) no Parque Estadual da Serra dos Pireneus, em Pirenópolis, Goiás. 
ro de morfotipos de galhas tanto no Cerrado (média de $22+0$ ) quanto na mata (média de $23+2)$, em comparação ao período chuvoso (Figura 3).

No presente estudo, verificou-se flutuação na riqueza de galhas de acordo com a mudança nos padrões sazonais (ANOVA: $\mathrm{F}_{3,4}=8,71, \mathrm{p}<0,05$, Figura 4). No início da estação seca, foi encontrada a maior riqueza de galhas nos dois habitats estudados (média de $23,5+1,5)$. No início da estação chuvosa, a riqueza de morfotipos caiu (média de $18+2$ ) até atingir o número mais baixo no final da estação chuvosa (média de 15 morfotipos por habitat). Com o início da estação seca, a riqueza de galhas voltou a subir, atingindo média de $21,5(+0,5)$ morfotipos de galhas por fitofisionomia.

\section{Discussão}

Os resultados obtidos neste estudo contrariam a hipótese do estresse nutricional, que prediz maior riqueza de galhadores em vegetações xéricas (Fernandes \& Price, 1988). $\mathrm{O}$ fato dos habitats mésicos possuírem maior número de árvores, com maior complexidade estrutural, pode ter influenciado a riqueza de galhadores neste ambiente (Gonçalves-Alvim

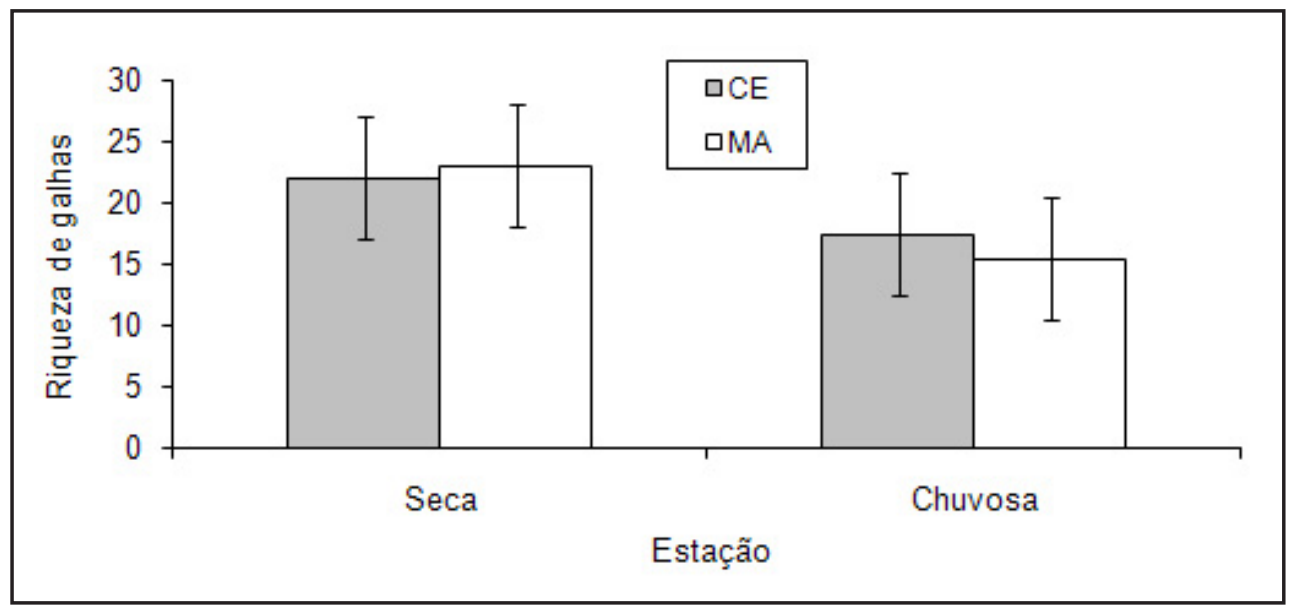

Figura 3 - Riqueza de morfotipos de galhas em habitats de Cerrado sensu stricto (CE) e mata semidecídua (MA) entre a estação seca e a estação chuvosa, no Parque Estadual da Serra dos Pireneus, em Pirenópolis, Goiás.

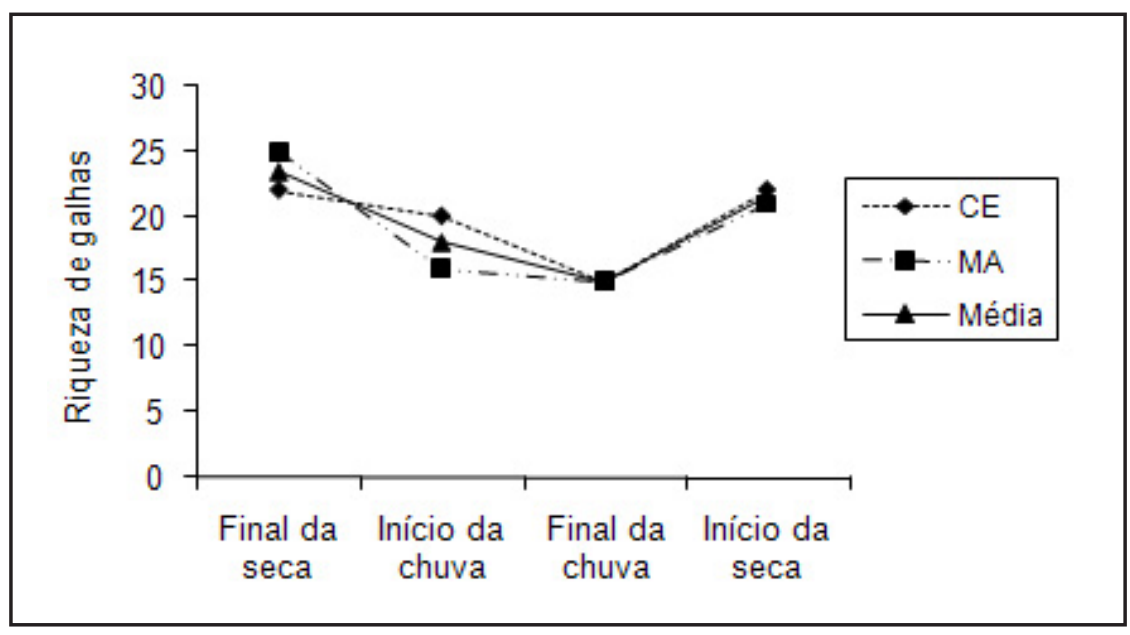

Figura 4 - Flutuação sazonal na riqueza de galhas em fitofisionomias de Cerrado sensu stricto (CE) e mata semidecídua (MA) no Parque Estadual da Serra dos Pireneus, Pirenópolis, Goiás. 
\& Fernandes, 2001). Para Collevatti \& Sperber (1997), à medida que aumentam, os parâmetros densidade, diâmetro e volume da copa parecem influenciar positivamente a riqueza de galhas. Plantas estruturalmente mais complexas representam maior disponibilidade de recursos (Lara et al., 2008) e de sítios de oviposição e também são mais facilmente avistadas pelos insetos (Collevatti \& Sperber, 1997), podendo abrigar grande diversidade de galhadores (Espírito-Santo et al., 2007). Esse fato pode ter sido responsável pela grande diversidade de galhas encontrada na fitofisionomia mésica. Entretanto, a hipótese da arquitetura das plantas hospedeiras não foi testada neste estudo.

Ferreira et al. (2007) estudaram a incidência de galhas em fitofisionomias de Cerrado sensu stricto e mata seca em Montes Claros, região norte de Minas Gerais. Os autores não encontraram diferenças significativas entre a riqueza de galhas nos dois habitats e atribuíram isto a pressões ambientais similares nas duas vegetações, como sazonalidade, pluviosidade e temperatura. De acordo com eles, tais fatores podem influenciar a riqueza e a abundância de galhas, uma vez que os insetos galhadores são intensamente afetados por condições intrínsecas das plantas hospedeiras, como sua presença ou ausência, e variações sazonais na qualidade dos recursos oferecidos pelas plantas (Fagundes et al., 2001).

A sazonalidade tem sido apontada na literatura como um fator determinante na diversidade de insetos herbívoros (Wolda, 1988). A riqueza e a abundância de insetos herbívoros de vida livre são bastante influenciadas por temperatura, precipitação e umidade (Kasenene \& Roininen, 1999). No caso dos galhadores, essa questão foi pouco abordada durante algum tempo, com o argumento de que os insetos estariam alheios às intempéries ambientais uma vez que se desenvolvem no interior das plantas hospedeiras (Fernandes et al., 1995). Contudo, em alguns estudos recentes, observou-se que a diversidade e a abundância dos galhadores podem variar de acordo com os padrões sazonais, principalmente em regiões climáticas com estações bem definidas (Dalbem \& Mendonça, 2006).
Durante a estação seca, a escassez de água provoca várias mudanças na fisiologia das plantas (Larcher, 2000). O estresse hídrico nas plantas inicia um complexo de respostas, começando com a própria percepção do estresse, desencadeando uma cascata de eventos moleculares, que é finalizada em vários níveis de respostas fisiológicas, metabólicas e de desenvolvimento, destacando-se o aumento da suscetibilidade ao ataque de herbívoros (Nepomuceno et al., 2001). As taxas de crescimento mais altas de muitas plantas do Cerrado ocorrem no período chuvoso, embora elas não cessem completamente seu desenvolvimento no período seco (Franco, 1998).

No final da estação seca, período em que foi encontrada a maior riqueza de galhas, tanto no habitat xérico quanto no mésico, o estresse hídrico provavelmente encontravase em seu nível mais extremo. A escassez de água e de nutrientes leva a modificações nos balanços hormonais e fisiológicos das plantas (Nepomuceno et al., 2001), resultando em modificações que comprometem os processos de defesa contra a herbivoria (Fernandes et al., 1995) e as tornam mais suscetíveis ao ataque de fitófagos (Christie \& Hochuli, 2005). A alta infestação encontrada durante os períodos de baixa pluviosidade seria explicada pelo desvio de metabólitos da planta hospedeira para a manutenção de suas atividades fisiológicas em detrimento das respostas direcionadas para a defesa contra a herbivoria (Ferreira et al., 2007). Desse modo, no período seco, as plantas estariam mais suscetíveis ao ataque dos galhadores e, consequentemente, a riqueza de morfotipos de galhas seria maior em comparação ao período chuvoso. Assim, as taxas de herbivoria em Roupala montana (Proteaceae) são maiores no período seco quando comparadas às dos meses chuvosos, podendo chegar até 16\% de perda foliar (Franco, 1998).

Em estudo recente, Araújo \& Santos (2009) investigaram os efeitos da sazonalidade na abundância de galhas de Cecidomyiidae (Diptera) em Piper arboreum (Piperaceae) em um fragmento de floresta semidecídua em Goiás. Os autores encontraram maior abundância de galhas no início do período chuvoso, que coincide com o brotamento da planta hospedeira. Por outro lado, Dalbem \& Men- 
donça (2006) verificaram que tanto a abundância quanto a riqueza de artrópodes galhadores no Rio Grande do Sul foram maiores no inverno, estação entre os meses de junho e setembro, que corresponde a período com baixa pluviosidade.

Os resultados do presente estudo apontam que padrões sazonais podem ser mais determinantes na diversidade de insetos galhadores do que o tipo fitofisionômico. Padrões sazonais em galhas e insetos galhadores ainda são pouco conhecidos e investigados. Estudos mais sistemáticos devem ser realizados para confirmar essa hipótese e demonstrar a real importância da sazonalidade na distribuição dos insetos galhadores.

\section{Agradecimentos}

Os autores agradecem ao Prof. Dr. Paulo de Marco Júnior (UFG) pelas críticas durante a realização do trabalho, aos revisores anônimos pelas sugestões ao manuscrito, ao PIBIC/ PRPPG/UFG pela concessão de bolsa de iniciação científica ao primeiro autor e ao Instituto de Ciências Biológicas da UFG pelo apoio logístico.

\section{REFERÊNCIAS}

Araújo, W. S. \& B. B. Santos. 2009. Efeitos da sazonalidade e do tamanho da planta hospedeira na abundância de galhas de Cecidomyiidae (Diptera) em Piper arboreum (Piperaceae). Rev. Bras. Ent. 53: 300-303.

Araújo, W. S., V. L. Gomes-Klein \& B. B. Santos. 2007. Galhas entomógenas associadas à vegetação do Parque Estadual da Serra dos Pireneus, Pirenópolis, Goiás, Brasil. Rev. Bras. de Biociênc. 5: 45-47.

Collevatti, R. G. \& C. F. Sperber. 1997. The gall maker Neopelma baccharidis Burck. (Homoptera: Psyllidae) on Baccharis dracunculifolia DC (Asteraceae): individual, local and regional patterns. Ann. Soc. Ent. Bras. 26: 45-53.

Christie, F. J. \& D. F. Hochuli. 2005. Elevated levels of herbivory in urban landscapes: are declines in tree health more than an edge effect? Ecol. Soc. 10: 1-10.
Cuevas-Reyes, P., M. Quesada, P. Hanson, R. Dirzo \& K. Oyama. 2004. Diversity of gall-inducing insects in a Mexican tropical dry forest: The importance of plant species richness, life-forms, host plant age and plant density. J. Ecol. 92: 707716.

Dalbem, R. V. \& M. S. Mendonça. 2006. Diversity of galling arthropods and host plants in a subtropical forest of Porto Alegre, Southern Brazil. Neotrop. Ent. 35: 616-624.

Espírito-Santo, M., F. S. Neves, F. AndradeNeto \& G. W. Fernandes. 2007. Plant architecture and meristem dynamics as the mechanisms determining the diversity of gall-inducing insects. Oecologia 153: 353-364.

Fagundes, M., M. L. Faria, G. W. Fernandes. 2001. Efeitos da distribuição de Baccharis dracunculifolia (Asteraceae) na abundância e no parasitismo de Neopelma baccharidis (Homoptera: Psyllidae). Unim. Cient. 1: 1-7.

Fernandes, G. W. \& P. W. Price. 1988. Biogeographical gradients in galling species richness: tests of hypotheses. Oecologia 76: 161-167.

Fernandes, G. W., A. S. Paula \& R. Loyola. 1995. Distribuição diferencial de insetos galhadores entre habitats e seu possível uso como bioindicadores. Vida Silv. Neotr. 4: 133-139.

Ferreira, M. F. M., P. M. S. Rodrigues, L. S. Araújo, C. H. P. Silva, J. B. Sampaio, B. G. Madeira. 2007. Comparação da incidência de galhas em duas formações florestais do Bioma Cerrado: cerrado sensu stricto e mata seca. Rev. Bras. Biociênc. 5: 36-38.

Fleck, T. \& C. R. Fonseca. 2007. Hipóteses sobre a riqueza de insetos galhadores: uma revisão considerando os níveis intra-específico e de comunidade. Neotrop. Biol. Conserv. 2: 36-45.

Franco, A. C. 1998. Seasonal patterns of gas exchange, water relations and growth of Roupala montana, an evergreen savanna species. Plant Ecol. 136: 69-76. 
Gonçalves-Alvim, S. J. \& G. W. Fernandes. 2001. Comunidades de insetos galhadores (Insecta) em diferentes fitofisionomias do cerrado em Minas Gerais. Rev. Bras. Zool. 18: 289-305.

Kasenene, J. M. \& H. Roininen. 1999. Seasonality of insect herbivory on the leaves of Neoboutonia macrocalyx in the Kibale National Park, Uganda. Afr. J. Ecol. 37: 61-68.

Lara, D. P., L. A. Oliveira, I. F. P. Azevedo, M. F. Xavier, F. A. O. Silveira, M. A. A. Carneiro \& G. W. Fernandes. 2008. Relationships between host plant architecture and gall abundance and survival. Rev. Bras. Ent. 52: 78-81.

Larcher, W. 2000. Ecofisiologia vegetal. São Carlos, RiMa, 531 p.

Mendonça, M. S. 2007. Plant diversity and galling arthropod diversity - searching for taxonomic patterns in an animal-plant interaction in the Neotropics. Bol. Soc. Arg. Bot. 42: 347-357.

Nepomuceno, A. L., N. Neumaier, J. R. B. Farias \& T. Oya. 2001. Tolerância à seca em plantas. Biotec. Ciênc. Desenv. 23: 12-18.
Price, P. W., G. W. Fernandes, A. C. F. Lara, J. Brawn, H. Barrios, M. G. Wright, S. P. Ribeiro \& N. Rothcliff. 1998. Global patterns in local number of insect galling species. J. Biog. 25: 581-591.

Ribeiro, J. S. \& B. M. T. Walter. 1998. Fitofisionomias do bioma Cerrado, p. 289-556. In: S. M. Sano \& S. P. Almeida. Cerrado: ambiente e flora. Brasília, DF, Embrapa/ CPAC.

Stone, G. N. \& K. Schönrogge. 2003. The adaptive significance of insect gall morphology. Trends Ecol. Evol. 18: 512-522.

Veldtman, R. \& M. A. McGeoch. 2003. Gallforming insect species richness along a non-scleromorphic vegetation rainfall gradient in South Africa: the importance of plant community composition. Aust. Ecol. 28: 1-13.

Wolda, H. 1988. Insect seasonality: Why? Ann. Rev. Ecol. Syst. 19: 1-18.

Data de recebimento: 30/IV/2009

Data de aceite: 20/XI/2009 